\title{
Old Evidence in the Development of Quantum Theory*
}

\author{
Molly Kao \\ Université de Montréal, Département de philosophie \\ C.P. 6128 succ. Centre-ville \\ Montreal, QC \\ H3C 3J7 \\ Canada \\ molly.kao@umontreal.ca
}

\begin{abstract}
In this paper, I evaluate Hartmann \& Fitelson's solution to the Bayesian problem of old evidence by applying it to an early stage in the development of quantum theory. I argue that this case study suggests that whether old evidence is anomalous or not affects its support for a hypothesis. I introduce and defend two formal assumptions to accommodate this idea. This analysis not only explicates an important historical example, but it also shows that the given solution captures the intuitive importance of "surprising" evidence that has previously been problematic in the context of old evidence.
\end{abstract}

*I am grateful to Samuel Fletcher, Richard Dawid and Yann Benétreau-Dupin for helpful comments and discussions on earlier versions of this paper. I would also like to thank two anonymous reviewers for their thoughtful suggestions. 


\section{Introduction}

The problem of old evidence (POE) in Bayesian epistemology has been discussed extensively, and it is generally understood that there are several issues that might bear this name $1^{1}$ In this paper, I will be concerned with the following type of situation. Consider the time shortly after a new theory has been formulated. We restrict our attention to a hypothesis $H$ in that theory and assume other information is contained in background $B$. Imagine that it is discovered that a previously known fact $E$ can be accounted for in some way by $H$, where $E$ was not used in the formulation of the theory. This newly discovered relation seems intuitively to contribute to the support for the theory. However, some have argued that in a Bayesian epistemological framework, it is difficult to account for why this should be, thus giving rise to the aforementioned POE. There have been several approaches to the solution of this problem, including (Garber 1983), (Eells 1990), and more recently, (Hartmann and Fitelson 2015).

Hartmann \& Fitelson call their solution a "more general and explanatory Bayesian approach" to POE (2015, 712). They present four formal conditions that, when satisfied, yield the result that learning about a relation between a newly developed theory and a previously observed phenomenon can raise one's credence in that theory. While the four conditions are meant to be intuitively plausible, it is left an open question whether it can be appropriately applied to any genuine scientific cases. However, if the goal of Bayesian approaches to philosophy of science is to reflect inferences made in scientific reasoning, I would argue that it is important that a proposed solution to POE be able to accurately explicate at least one known historical case of old evidence. In general, discussions rooted in scientific cases help to demonstrate the significance of Bayesian epistemology beyond its role as a purely logical exercise. While this latter is indisputably important, we can gain understanding of the domain of application of formal analyses when new developments in the framework are anchored in historical or contemporary scientific practice. Indeed, such case studies can contribute to both the descriptive and normative aspects of Bayesian approaches. It is clear that an accurate historical explication will contribute to the idea that this solution is genuinely descriptive, which is what Hartmann \& Fitelson seem to have in mind in their discussion. Of course, there also exists a potential normative element. A previously successful case of reasoning that conforms to the Bayesian analysis gives us some reason to think that this analysis could be applicable in other contexts.

In this paper, I provide an evaluation of Hartmann \& Fitelson's solution by investigating its ability to explicate examples of old evidence in the early stages of the development of quantum theory. More specifically, I consider how a postulate about the discrete nature of energy, i.e. a quantum postulate, was used to account for the previously known results of several experiments. I begin by showing how this might have worked in Einstein's explanation of the specific heat of diamond, and Bohr's old quantum theory with respect to Balmer's formula for

\footnotetext{
${ }^{1}$ For instance, see (Garber 1983), (Jeffrey 1983), (Christensen 1999).
} 
the spectrum of hydrogen. This discussion shows that Hartmann \& Fitelson's solution provides an appropriate framework for the explication of these cases of old evidence in the sense that the four formal conditions they present are satisfied.

The application of a quantum postulate in these domains is then contrasted with Einstein's use of light quanta to explain Stokes's rule in order to highlight the significance of the fact that a phenomenon being explained is anomalous, where this indicates that the phenomenon seems to be in direct conflict with dominant theories. I argue that an explication of old evidence that aims to accurately formalize a reasonable agent's thought processes should reflect this fact. The framework of the solution, as it stands, does not fully account for the cases in question. That is, the consideration of the historical facts necessitates the introduction of further assumptions to accurately capture the situation. I thus propose and defend an addition to the given framework in the form of two new formal assumptions, which I claim overcomes the identified shortcomings such that their addition results in an appropriate explication of the support provided to the quantum postulate.

This analysis yields some interesting results. First, it provides an explicit demonstration that Hartmann \& Fitelson's solution can explicate some genuine cases of old evidence. Second, it shows how we can capture an intuitive result from Bayesian confirmation theory that is difficult to account for in the case of old evidence. Specifically, we see in detail how the more surprising of two consequences of a hypothesis may better support that hypothesis, even when an agent's credence in both those consequences is equal to one. Thus, we are able to give a descriptive account of this feature, where we need not abstract from an agent's actual state of knowledge about how likely a piece of evidence is to conclude that a surprising prediction is more confirmatory.

The rest of the paper proceeds as follows. In the next section, I briefly describe the POE and Hartmann \& Fitelson's solution. Section 3 provides an overview of two of the historical phenomena in question, and shows how they conform to the framework of the given solution. In section 4, I present an example that has some contrasting elements with the previous two cases. I thus discuss the implications of this case in section 5. Specifically, I propose two further restrictions on an agent's credences in cases where old evidence is operative, then I show how these additions allow us to see the relation between old evidence and the value of surprising evidence.

\section{Old Evidence}

In formulating the POE, we consider the impact of evidence $E$ on hypothesis $H$ in terms of the simple conditionalization rule. Thus, upon learning a piece of evidence $E$, an agent's degree of belief in $H$ should be revised according to the conditionalization formula, $\operatorname{Pr}(H \mid E)=$ $[\operatorname{Pr}(H) \cdot \operatorname{Pr}(E \mid H)] / \operatorname{Pr}(E)$, where all quantities are understood to be conditional on background information $B$. The problem is that for any evidence $E$ that is already known, both 
$\operatorname{Pr}(E)$ and $\operatorname{Pr}(E \mid H)$ have the value 1 due to the fact that evidence $E$ is absorbed into the background $B$ term implicitly present in both values. Thus, $\operatorname{Pr}(H \mid E)$ has the same value as $\operatorname{Pr}(H)$, and so it seems that any fact that is already known at the time that we are considering a new hypothesis $H$ cannot raise our credence in $H$. Yet, both intuitively and historically, it seems that such old evidence can provide support for a new hypothesis. Classic examples include the increased confidence in Einstein's theory of general relativity due to its explanation of the previously known precession of Mercury's perihelion, and the support provided to Newton's argument for universal gravitation from previous observation of Kepler's laws. In both these cases, despite the fact that the evidence statements were known before the time that the theories were formulated, we tend to think these evidence statements should raise one's credence in the theories, represented symbolically by $\operatorname{Pr}(H \mid E \& B)>\operatorname{Pr}(H \mid B)$.

There have been several suggested solutions to the problem. One class, demonstrated by (Howson 1991), eliminates $E$ from the background information so that probabilities $P(H)$ and $P(H \mid E)$ are relativized to knowledge $B-\{E\}$. However, another class of solutions accepts that $E$ is included in $B$. In this case, the problem is rooted in the assumption that an agent is perfectly logically omniscient and is thus aware of the relations between $H$ and $E$ as soon as a theory which contains $H$ is formulated. If that is so, there is no new fact about the relation between $E$ and $H$ that can change the agent's credence in $H$. Thus, (Garber 1983), among others, discusses solutions that eliminate the assumption of logical omniscience on the grounds that it is an unrealistic representation of any agent's degrees of belief. This type of solution is now often referred to as a Garber-style solution to POE, and it is the strategy employed by Hartmann and Fitelson (2015).

Their solution relies on the claim that what is learned by an agent is that some relation obtains between a hypothesis $H$ and evidence $E$, perhaps a logical one. It is the discovery of this fact that provides support to the hypothesis ${ }^{2}$ Hartmann \& Fitelson show that the satisfaction of four formal conditions yields the result that learning that $H$ accounts for $E$ contributes to the support for $H$.

Take statements $X$ and $Y$, which have to do with whether a hypothesis $H$ adequately accounts for the evidence in question $]^{3} X$ is the statement that hypothesis $H$ adequately explains or accounts for evidence $E$. $Y$ is the statement that $H^{\prime}$ 's best competitor $\left(H^{\prime}\right)$ adequately explains or accounts for evidence $E$. The solution requires four ordinal assumptions for the support to go through:

1. $\operatorname{Pr}(H \mid X \& \neg Y)>\operatorname{Pr}(H \mid \neg X \& \neg Y)$

2. $\operatorname{Pr}(H \mid X \& \neg Y)>\operatorname{Pr}(H \mid \neg X \& Y)$

\footnotetext{
${ }^{2}$ Hartmann \& Fitelson discuss their solution in terms of the confirmation of a theory $T$ rather than a hypothesis. Since I am interested in the role of the quantum postulate specifically, I take it to be the hypothesis whose support is in question, and other assumptions to be included in the background information.

${ }^{3}$ The notion "accounts for" is deliberately vague: according to Hartmann \& Fitelson, it can be satisfied by relations such as logical entailment and explanation, among other possibilities.
} 
Copyright Philosophy of Science 2017

Preprint (not copyedited or formatted)

Please use DOI when citing or quoting

3. $\operatorname{Pr}(H \mid X \& Y)>\operatorname{Pr}(H \mid \neg X \& Y)$

4. $\operatorname{Pr}(H \mid X \& Y) \geq \operatorname{Pr}(H \mid \neg X \& \neg Y)$

I will not explicate these in detail here as their interpretation in specific contexts will be the focus of later sections. The idea that these assumptions are intuitively plausible is defended in the original paper. For now, we can note that each of them requires a certain ordinal ranking of an agent's credence in hypothesis $H$ that depends on the various possibilities of $H$ and $H^{\prime}$ accounting for evidence $E$. For instance, the first condition can be understood as saying that in a case where $H$ 's best competitor does not account for $E$ but $H$ does, one should have higher credence in $H$ than the case where neither $H$ nor its best competitor account for $E$.

The proof proceeds by noting that these ordinal assumptions lead to the result that $\operatorname{Pr}(H \mid X)>$ $\operatorname{Pr}(H)$. To see this, define the following probabilities in this way: $a=\operatorname{Pr}(H \mid X \& \neg Y), b=$ $\operatorname{Pr}(H \mid X \& Y), c=\operatorname{Pr}(H \mid \neg X \& \neg Y), d=\operatorname{Pr}(H \mid \neg X \& Y)$. Also, let $x=\operatorname{Pr}(\neg Y \mid X), y=$ $\operatorname{Pr}(\neg Y \mid \neg X)$. The ordinal assumptions above can then be written as follows:

1. $a>c$

2. $a>d$

3. $b>d$

4. $b \geq c$.

As a result of these inequalities,

$$
a x+b(1-x)>c y+d(1-y) .
$$

This in turn entails that $\operatorname{Pr}(H \mid X)>\operatorname{Pr}(H)$, i.e. that learning $X$ should raise an agent's degree of belief in hypothesis $H$.

\section{Old Evidence in Quantum Theory}

As mentioned previously, my goal is to evaluate Hartmann \& Fitelson's solution by assessing its ability to explicate a historical example of old evidence. In what follows, I consider how invoking a "quantum postulate" about the discretization of certain quantities made it possible to account for experimental results that previously had no explanation. The postulate I propose can be stated as follows: "There is a universal, nonzero parameter $h$ with the dimension of action (energy - time) that can be used to impose a quantization condition on quantities that were previously considered to be continuous." This postulate is a way of expressing the idea of discretization of energy that was first suggested by Planck in 1900 to explain the emission spectrum of blackbody radiation. 


\subsection{Specific heat of diamond}

At the beginning of the twentieth century, a problem with specific heats loomed over parts of the scientific community. The specific heat of a substance is the amount of heat required to raise the temperature of a unit mass of that substance by one degree Kelvin. Lord Kelvin, in a lecture to the Royal Society in 1900 described this problem as a "cloud which has obscured the brilliance of the molecular theory of heat and light during the last quarter of the nineteenth century," (William Thomson 1901, 527). Despite many successes of the atomic-molecular theory of gases, the combination of this conception of matter with the Maxwell-Boltzmann doctrine of the equipartition of energy demanded by classical physics yielded mixed results in the prediction of specific heats of various substances. Its application in the Dulong-Petit law successfully predicted the specific heats of certain crystalline structures at high temperatures, but the same law yielded values that were significantly higher than those observed for substances such as diamond at room temperature $4^{4}$ Thus, the observation in question here is the low value for the specific heat of diamond at room temperature.

Before 1906, no one had considered the idea that quantum concepts should be incorporated into an analysis of specific heats, or that Planck's work on blackbody radiation could be relevant for these phenomena. Yet, Einstein motivated his treatment of diamond by arguing that if Planck's quantum postulate from blackbody radiation goes to the "root of the matter," then we might well expect other anomalous phenomena in the context of heat and radiation, and that such anomalies could potentially be addressed by similar quantum postulates (Einstein 1989. 219).

Einstein proposed that the idea of quantization by way of the parameter $h$ be applied to the energy of atoms of solids. He thus required that the energy of an atom oscillating at frequency $\nu$ take on values infinitesimally close to the discrete values $n h \nu$, where $n=0,1,2 \ldots$ Using this conjecture in combination with a canonical distribution to describe a system, he calculated the mean energy of an oscillator with frequency $\nu$. By making the simplifying assumption that all the atomic vibrations were independent and of the same frequency $\nu$, Einstein was able to calculate the average energy of one oscillator (Einstein 1907, 186). The energy of one mole of such a solid would be

$$
E=3 R \frac{h \nu / k}{e^{\frac{h \nu}{k T}}-1},
$$

and the specific heat can be calculated by differentiating the energy with respect to temperature.

\footnotetext{
${ }^{4}$ The Dulong-Petit law provided the molar specific heat capacity of a substance as a constant with units Joules/Kelvin. This constant is now expressed as $3 R$, where $R$ is the universal gas constant. We now know that these divergences between predictions and observations occur because of quantum effects at relatively low temperatures: broadly, some degrees of freedom require a certain minimum temperature before they are activated and can store energy.
} 
Note that as $k T / h \nu$ approaches zero, $h \nu / k T$ grows large, and the expression for $E$ approaches zero as well. In the limit as $k T / h \nu$ goes to 1 and larger, the expression takes the value of $3 R$. As Klein explains, "If the specific heat is plotted as a function of temperature, or rather of $(T k / h \nu)$, one obtains a curve that rises smoothly and monotonically from zero at the origin and approaches the equipartition value, $3 R$, asymptotically, when $(T k / h \nu)$ becomes large" (Klein 1965, 176).

This relation predicts that the specific heats of solids that conform reasonably well to Einstein's assumptions should be very low at low temperatures, and will approach the value predicted by classical theories at much higher temperatures. The contradictions between the experimental observations and theoretical predictions based on equipartition occurred primarily among lighter atoms, which could be expected to vibrate at higher frequencies, i.e. higher values of $\nu$, at which the value of $E$ approaches 0 . Einstein's quantized treatment thus fits well with these observations since they would be expected not to conform to the equipartition predictions, but to be much lower. In particular, according to the equipartition theorem and the Dulong-Petit law, the molar specific heat of diamond would be 3R. However, the observed value is approximately $0.735 \mathrm{R}$. With their relatively low atomic weights, atoms of carbon could be expected to have a higher oscillation frequency according to Einstein's assumptions, and thus a lower specific heat than the value predicted by the Dulong-Petit law.

We can see how Einstein's analysis was able to turn the specific heat of diamond at room temperature into 'old evidence' for the quantum postulate. Before this account, there was no explanation for the observed value of this quantity. The quantum postulate put forth above served as Einstein's starting point. The hypothesis that there was some discretization condition on the energy of the molecules allowed him to describe the specific heats of certain solids in general. The previously observed low value of the specific heat of diamond was then accounted for by this description.

\subsection{Balmer's formula}

The second instance of old evidence I will be considering is the expression of Balmer's formula for the spectrum of hydrogen, and its account in terms of Bohr's old quantum theory as presented in (Bohr 1913). The primary goal of this work was to provide a preliminary theory of the structure of the atom. The assumption Bohr uses in his model of the hydrogen atom takes the fundamental idea of quantization directly from Planck's work on radiation phenomena, but with a significantly different interpretation. He shows how the application of assumptions found in Planck's theory of radiation to ideas on the atomic structure of hydrogen results in an account of how electrons might be bound to a positive nucleus in stable states. He considers the behaviour of energy in the context of an atomic system, and describes the emission process as taking place in quantized amounts, dependent on the parameter $h$. Using this conjecture along with his elementary atomic model, Bohr was able to account for the Balmer formula, which described the discrete spectral lines observed when hydrogen gas is heated. Our focus 
here will be on this latter fact.

The Balmer formula can be expressed in a generalized form known as the Rydberg formula,

$$
\nu=R\left(\frac{1}{\tau_{2}^{2}}-\frac{1}{\tau_{1}^{2}}\right)
$$

where $R$ is Rydberg's constant, and $\tau_{1}$ and $\tau_{2}$ are integers greater than or equal to 1 such that $\tau_{1}<\tau_{2}$. These precise spectral lines could not be explained on the classical theory. Indeed, one might say they were doubly anomalous. One would first expect, according to classical electromagnetic theory, that an electron orbiting a nucleus would emit energy proportional to its rotational frequency, and that this frequency would change continuously as the energy is emitted, thus yielding energy of a continuous spectrum. Yet, the emitted radiation was of an integral number of specific frequencies, as manifested in a number of discrete lines on the spectrum. Furthermore, even if one were to accept the existence of stable states in the atom corresponding to these discrete spectral lines, classical physics predicts that the lines of the higher harmonics should be sums of the fundamental frequencies, whereas the observed spectral lines were expressed by Ritz in his 'combination principle' as differences between the harmonics (Jammer 1966, 69). This made Bohr's accomplishment even more significant.

Although Balmer's formula had been identified as accurately describing the observed spectral lines, there was no theoretical account of how and why these lines were produced until Bohr put forth his model of the hydrogen atom. On this model, electrons moved in stationary orbits that were determined in part by the parameter $h$. Upon transition from one energy level to another, an electron would emit a discrete amount of energy, $h \nu$. Bohr considered the case of a hydrogen atom, which was generally accepted as a system in which a single electron rotated around a positive nucleus of charge $e$. According to his earlier calculations, the binding of an electron to a positive nucleus in a transition to that stable state would result in certain amount of emitted energy.

To express the amount of energy emitted by the system when transitioning from a state $\tau_{1}$ to another state $\tau_{2}$, we have

$$
W_{\tau_{2}}-W_{\tau_{1}}=\frac{2 \pi^{2} m e^{4}}{h^{2}} \cdot\left(\frac{1}{\tau_{2}^{2}}-\frac{1}{\tau_{1}^{2}}\right) .
$$

where $m$ and $e$ are the mass and charge of an electron. By comparing with Equation 3 we see that this is simply an expression of the formula put forth by Balmer, where $R$ is replaced by more specific constants.

Thus, we see that Bohr was able to use a quantum postulate to construct an atomic model that was able to account for a previously observed phenomenon. 5 By using a quantum postulate

\footnotetext{
${ }^{5}$ Here, I take it that Bohr did not construct his quantum theory with the Balmer formula in mind, in the sense that this observation does not seem to be used directly to determine the equations describing the atomic system. Instead, he justifies and explains his assumptions in terms of the need to define stable states for an atom of
} 
Copyright Philosophy of Science 2017

Preprint (not copyedited or formatted)

Please use DOI when citing or quoting

to formulate a model of the hydrogen atom, and then using this model to calculate correct values for the previously observed spectral lines of hydrogen that had no explanation, Bohr turned those observations into 'old evidence' for the quantum postulate.

\subsection{Bayesian analysis}

Although the conditions given by Hartmann \& Fitelson listed above are meant to be plausible enough to apply in any case, it is useful to consider what they mean in our particular context. We have seen that the conditions are all based on comparative probabilities of a hypothesis $H$, conditional on various combinations of ways that a phenomenon might be accounted for by $H$ or by its best alternative $H^{\prime}$. Thus, in this context, we should read $H, X$ and $Y$ as expressing claims about the quantum postulate in relation to the phenomena of specific heat of diamond or Balmer's formula. Specifically, $X$ and $Y$ are the following. $X$ : "QP adequately explains (or accounts for) $E$, the measured specific heat of diamond near room temperature." Y: "Classical physics, with its assumption of continuity for the quantities in question, adequately explains (or accounts for) $E$, the measured specific heat of diamond." While I conduct my analysis in terms of specific heats, the same points apply, mutatis mutandis, to the account of Balmer's formula by Bohr's old quantum theory.

Recall that Hartmann \& Fitelson's result requires the acceptance of four conditions, as outlined in section 2. In our case, the first condition, which can be written $\operatorname{Pr}(Q P \mid X \& \neg Y)>$ $\operatorname{Pr}(Q P \mid \neg X \& \neg Y)$, states that the quantum postulate is more likely if it is able to account for the specific heat of diamond and classical physics is not, than if neither it nor classical physics with its assumption of continuity could account for this phenomenon. Historically, it was this ability to explain the phenomenon that rendered it a promising postulate for many scientists, so the ordinal ranking is reasonable. The second condition says that QP is more likely if it accounts for the specific heat of diamond while classical theory does not, as opposed to a situation where classical theory could account for it and a quantum postulate could not. This reflects the idea that if classical theory could account for the specific heat values and QP could not, there would have been no reason to think of QP as being promising in relation to this phenomenon. Indeed, this condition was expressly satisfied historically, since it did indeed seem that classical physics could not account for the specific heat of diamond, while a quantum postulate could.

The third condition says that QP is less likely in the case that only classical theory could explain the specific heat than in the case where both QP and classical theory are possible explanations of the specific heat. This seems reasonable, since even if classical theory were also able to account for the specific heat, the ability of QP to do so as well seems like it would

hydrogen, and the justification of these assumptions is that they yield values that correspond to independently calculated linear dimensions of the atom, and ionization-potentials. Thank you to an anonymous reviewer for this point. 
render QP more promising than if it could not. One might claim that if classical theory could account for the specific heat of diamond, then scientists would not have been concerned with a quantum postulate in this context at all. While this may very well be true, this condition simply ranks credence in the quantum postulate as being higher if it accounts for the specific heat than if it does not; the actual values may well be very low. The last condition states that QP is at least as likely if both it and its competitor explain the specific heat than if no theory at all does so. Again, this seems reasonable: imagine that at the same time that QP was shown to be able to account for the specific heat, a plausible way of adjusting classical theories to account for the phenomenon was also suggested. One might not think that QP is extremely likely in this case, but its ability to account for the phenomenon should not make it less likely than previously.

Given these assumptions, Hartmann \& Fitelson show that $\operatorname{Pr}(Q P \mid X)>\operatorname{Pr}(Q P)$. This means that, if one were to learn that QP accounts for the specific heat of diamond, then this raises one's degree of belief in QP. Similarly, learning that QP accounts for Balmer's formula raises one's degree of belief in QP. This particular solution is fitting for a historical reconstruction for other reasons. The first is the use of a quite general idea that a theory "accounts for" a phenomenon. Hartmann \& Fitelson purposely allow for an open interpretation as to what this means, which makes it particularly suitable for an explication of the role of the quantum postulate. Interpretations of what QP actually represented physically were quite varied, but this fact is not a problem in this framework. The second reason is the contention that what is being learned that contributes to the support for QP is the fact that it accounts for a phenomenon that was previously known. This certainly tracks what scientists seemed to be motivated by historically, since the interest always arose in response to a demonstration that the quantum postulate could account for the phenomena. We thus see how Hartmann \& Fitelson's solution provides an accurate description of historical results for these phenomena.

\section{A contrast case}

In Section 3, I discussed two examples in which a quantum postulate was used to account for experimental results that were known prior to the application of that postulate and indeed, prior to the first articulation of the postulate. In both cases, the fact that the phenomena were explicable in terms of the quantum postulate seemed to provide that postulate with some support. That is, the fact that one could apply a discretization condition to certain quantities in these two contexts and thus account for previously unexplained phenomena, seemed to make the general strategy of using the parameter $h$ to discretize quantities in different contexts more plausible. This can be inferred by noting that the quantum postulate was pursued further in the two cases treated. For instance, Nernst reported on his improved experiments on the specific heat of diamond (see Nernst 1910), citing Einstein's explanation of the anomalous observations, and published an article "On the theory of specific heat and the application of the theory of energy quanta to physical-chemical problems in general" (1911), in which he used the idea of quanta 
to guide general elements of his research. The striking though limited success of Bohr's old quantum theory led to the more than decade-long pursuit of a better theory of the atom that incorporated a quantization condition.

However, we can contrast these with another case of explanation of a previously known phenomenon, namely, Einstein's use of a quantum postulate to explain Stokes's rule (Einstein 1905), which was an experimentally well-confirmed result dating back to 1852 . This explanation also arose in a context where a theory was put forth based on a quantum postulate, namely, Einstein's light quanta theory. Although inspired by a quantum postulate, Einstein argues for the hypothesis of light quanta based solely on an analogy between the entropy of a system of radiation and that in a gaseous system. The bulk of the paper is devoted to the foregoing analysis. However, using this light quanta postulate, Einstein was then able to provide an account of three previously observed phenomena, including Stokes's rule.

Einstein considered observations of photoluminescence, where monochromatic light is changed to light of a different frequency when being absorbed and re-emitted by various forms of matter. He assumed that the original and the changed light consist of energy quanta of size $h \nu$, and that one incoming light quantum was responsible for one outgoing light quantum. By conservation of energy, he reasoned that the final energy of a light quantum would have to be less than that of an initial light quantum or, symbolically, $\frac{R}{k N} h \nu_{2} \leq \frac{R}{k N} h \nu_{1}$. This is simply an expression of Stokes's rule that incorporates Planck's constant.

Despite the fact that Einstein's explanation of Stokes's rule provided an account of a phenomenon that was previously known, scientists did not react with any great enthusiasm. There was little to no uptake of the idea of light quanta that Einstein had introduced. One might argue that this is simply because most found the idea of light quanta too radical, and that such a hypothesis would need far more direct experimental evidence in order to be seriously considered by many people. Yet, it is worth considering why this was such a contrast to the reception of Einstein's quantum postulate as applied to specific heats. Even though early applications of the postulate to specific heats could not be tested in experimentally precise ways, it was received as an extremely promising explanation of the phenomenon in question and triggered several rounds of new experiments almost immediately.

Based on these reactions, it seems that this was because the use of a quantum postulate provided an account of phenomena that were not only previously known and inexplicable by other means, but that indeed seemed to be in direct conflict with the dominant theories of the time. Such a feature makes the need for an account of the phenomenon much more urgent, and I claim this was an operative feature of the cases of specific heats and spectral lines discussed above. This can be contrasted with the case of Stokes's rule: while it is true that at the time of Einstein's 1905 paper this rule was not explicable in terms of classical theories of light and energy, it was not the case that the results were widely recognised as being an insurmountable challenge for those theories. Instead, it seemed reasonable that one would be able to develop an account of this phenomenon in the classical framework by taking into account some previously unknown special conditions. Thus, any potential changes could be seen more as amendments 
Copyright Philosophy of Science 2017

Preprint (not copyedited or formatted)

Please use DOI when citing or quoting

to classical theory, rather than an outright upheaval. It is also worth noting that in all three examples I have discussed, the old evidence in question can be considered instances of "novel confirmation" in the sense that the theories incorporating a quantum postulate did not try to accommodate the phenomena in the development process. The issue is thus whether an account of how old evidence supports a hypothesis can plausibly accommodate this apparent difference, where accounting for strongly anomalous phenomena seems to provide better support for a hypothesis than accounting for one that is not viewed as anomalous.

\section{Implications}

In section 3.3 I considered this case study only in terms explicitly presented by Hartmann \& Fitelson. However, we can extend the analysis to shed further light on the significance of old evidence, as well as its relations with other features of Bayesian epistemology. I will present two features that emerge from historical considerations, namely the role of a surprising application of a hypothesis, and the role of anomalous evidence. I will show that taking these into account in our explication reveals relations to the feature of surprising evidence in Bayesian confirmation.

\subsection{Surprising Application}

First, consider the difference between $\operatorname{Pr}(Q P \mid X)$ and $\operatorname{Pr}(Q P)$, which can be written as follows:

$$
\operatorname{Pr}(Q P \mid X)-\operatorname{Pr}(Q P)=\operatorname{Pr}(\neg X)[\operatorname{Pr}(Q P \mid X)-\operatorname{Pr}(Q P \mid \neg X)] .
$$

Let us suppose that the conditional probabilities for $Q P$ on $X$ and $\neg X$ are fixed, regardless of the value of $\operatorname{Pr}(X)$. That is, we suppose that regardless of how likely we think it is that the quantum postulate accounts for the specific heat of diamond, the fact of its accounting for this phenomenon has a fixed relation with our credence in the postulate itself. Then the amount to which one's degree of belief in $Q P$ is raised is proportional to $\operatorname{Pr}(\neg X)$. In other words, it is a decreasing function of $\operatorname{Pr}(X)$, one's prior belief that the hypothesis accounts for the phenomenon. This means that if prior to learning definitely that QP accounts for the specific heat, one already thought that this was likely to be the case, then learning this fact does not add as much to the support for QP as if one were more skeptical of the idea that QP could account for this phenomenon.

In our particular case, it does seem as though it would be relatively surprising that QP was able to account for the specific heat of diamond. This is, in part, due to the fact that the QP itself was not obviously reconcilable with certain successful aspects of classical physics. What made it even more surprising was its application to a context that seemed unrelated to any of the contexts in which QP was previously successful, i.e. to a theory of energy in solids 
Copyright Philosophy of Science 2017

Preprint (not copyedited or formatted)

Please use DOI when citing or quoting

rather than the context of energy transmission between radiation and matter. Thus, it seems reasonable that Einstein's demonstration that a quantum postulate could account for aspects of the specific heat of diamond played a significant role in raising scientists' degree of belief in this hypothesis, given its very surprising nature. It is worth noting here that this is different from the idea of the evidence itself being surprising : what is in question is not the result itself, but the ability of a particular (quantum) hypothesis to account for the evidence in question.

\subsection{Anomalous Evidence}

I now turn to the significance of anomalous phenomena I discussed in the previous section. While Hartmann \& Fitelson do not discuss this issue, I drew attention to the fact that historically, it seems that learning that a hypothesis accounts for a previously known phenomenon is not always taken to be strong evidence for that hypothesis. The cases where we put the most weight on old evidence are those where it was believed that this evidence was not accounted for by any previous theory. In the classic example of general relativity being able to account for the precession of the perihelion of Mercury, it was intuitively seen as highly significant that Einstein's theory could account for this phenomenon, whereas being able to account for the observations that Newton's theory also covered would be merely expected of any competing theory. In the cases of both specific heats of diamond at room temperature and the spectral lines expressed by Balmer's formula, it seemed significant that classical theory yielded predictions that were in direct conflict with observations of these phenomena.

I take it that this fact can serve as a guide to conditions that a solution to old evidence should satisfy. We can express this as the condition that an agent's credence in a hypothesis should be boosted the most in cases where it can account for a phenomenon that previous theories could not account for. In other words, the support for $H$ (or in our case QP) should vary with an agent's credence in $\operatorname{Pr}(Y)$, i.e. how likely we think that the classical theory can account for the phenomena in question. If we examine Hartmann \& Fitelson's framework, we can infer that such a correlation holds in the case where two separate conditions are satisfied: first, the relation $a-c>b-d$ holds, and second, $\operatorname{Pr}(Y)$ and $\operatorname{Pr}(X)$ are independent. When the latter condition holds, $\operatorname{Pr}(\neg Y \mid X)=\operatorname{Pr}(\neg Y \mid \neg X)$, so that $x=y$. When this is true, we see that Equation 1 becomes $a x+b(1-x)>c x+d(1-x)$. If the first condition holds, the difference between the two sides of the equation varies with $x$, as desired. I argue that these conditions are satisfied in cases we would intuitively consider accounting for old evidence to have contributed to an agent's credence in a hypothesis.

First consider the assumption that $\operatorname{Pr}(Y)$ and $\operatorname{Pr}(X)$ are independent. $\operatorname{Pr}(Y)$ refers to the credence an agent has in the proposition that classical theory accounts for the specific heat of diamond at room temperature, while $\operatorname{Pr}(X)$ is the credence an agent has in the proposition that a quantum postulate accounts for the specific heat of diamond at room temperature. Before the quantum postulate was ever applied to this phenomenon, it was known that classical theory could not account for the specific heat, and so $\operatorname{Pr}(Y)$ was very low. The subsequent use of the 
quantum postulate had no bearing on this fact. Thus, it is reasonable to think that at least in this case, these two probabilities are independent. Similarly, in the case of Balmer's formula, Bohr's introduction of old quantum theory to provide an account of this formula did not change a low credence in the proposition that classical theory could account for these spectral lines.

One might be concerned about this condition since the relations between $\operatorname{Pr}(X)$ and $\operatorname{Pr}(Y)$ are features of the probability space. Thus, there is perhaps simply a fact of the matter that a low credence in $Y$ would affect the credence in $X$, and so we cannot assume independence. However, in the situation we are considering, we have explicitly eliminated the assumption of logical omniscience of the agent. This means that we can assign values to $\operatorname{Pr}(X)$ and $\operatorname{Pr}(Y)$ that make sense for an agent in that context, and at the time point that we are considering, it is reasonable to consider the two credences as being independent. After all, even if it was known that the classical theories did not account for the phenomena, the quantum postulate modifies a very specific aspect of classical theories. Presumably, there are countless other ways to modify classical theory that would result in hypotheses that would not account for the specific heat. Of course, this independence condition will not always hold: for instance, after the formulation of Bohr's correspondence rule, the ability of $Q P$ to account for phenomena in certain domains would be known to depend on the ability of certain classical descriptions of phenomena. Such relations would have to be evaluated with the individual cases in mind.

Now consider the condition that $a-c>b-d$. This is the same as a condition that $a-b>c-d$. The difference $a-b$ is the difference between $\operatorname{Pr}(H \mid X \& \neg Y)$ and $\operatorname{Pr}(H \mid X \& Y)$. The difference between $c-d$ is the difference between $\operatorname{Pr}(H \mid \neg X \& \neg Y)$ and $\operatorname{Pr}(H \mid \neg X \& Y)$. I claim that it is reasonable to expect the first probability difference to be larger than the second.

This is because in the first case, we are assuming that $H$ accounts for a phenomenon, and the difference relies on whether there is another hypothesis that does the same. In the second case, we are evaluating the probability of a hypothesis conditional on the fact that the hypothesis does not account for the phenomenon in question. I would argue that the presence of an alternative explanation is more meaningful in the first case. In the first case, when a hypothesis accounts for evidence, a reasonable thing to ask is whether there are alternative explanations for the phenomenon. In our particular example, this expresses the idea that the quantum postulate's ability to account for the specific heat of diamond when classical theory could not was significant. However, if a hypothesis does not account for the evidence in question, the latter fact seems to be less important, in the sense that our credence in $H$ was likely to be lower in the first place.

Imagine for instance that we are assigning a credence to the old quantum theory, conditional on the fact that it does not account for the observation of certain spectral lines, such as those of helium. This failure to account for a given phenomenon seems to render our credence in that postulate quite low. This is still true when the best possible competitor to $H$ also fails to explain the phenomenon. Imagine now that a theory is developed that could account for the spectral lines. It is true that this might lower our credence in old quantum theory even more, but given the latter's failure to account for the phenomenon in which we were originally interested, 
there is less of a margin by which $\operatorname{Pr}(H)$ may be reduced. In our particular case, credence in a quantum postulate that did not explain the specific heat would be low, regardless of whether classical theory could account for specific heat or not. Therefore, we would expect that the difference between $c$ and $d$ is smaller than the difference between $a$ and $b$.

When the two above conditions are satisfied, the difference between $\operatorname{Pr}(H \mid X)$ and $\operatorname{Pr}(H)$ will vary with $\operatorname{Pr}(\neg Y)$. Particular numbers will depend of course on the priors an agent assigns to all the conditional probabilities. However, this dependence captures the fact that accounting for something like specific heat, where $\operatorname{Pr}(\neg Y)$ is very high, will provide relatively more support for QP than accounting for a phenomenon like Stokes's rule, where $\operatorname{Pr}(\neg Y)$ is considerably lower.

\subsection{Discussion}

Of course, the formal conditions that I have introduced may not always be satisfied in historical cases of old evidence. For instance, it might be the case that an agent knows the precise ways in which $\operatorname{Pr}(Y)$ and $\operatorname{Pr}(X)$ are probabilistically related, and so independence between those will not hold. This would simply mean that the feature I identify here does not play a role in the explication of the support of old evidence. Furthermore, this is not to claim that a phenomenon such as Stokes's rule does not provide any support at all to the quantum postulate, but merely to claim that the support is less in comparison to the other phenomena.

Finally, we can see the significance of these results for Bayesian approaches in general due to an important reflection of a feature that is a standard result of Bayesian confirmation theory. We know that if evidence $E$ is a logical consequence of hypothesis $H$, the support $E$ provides to $H$ is higher when it is more surprising or unexpected (i.e. when $\operatorname{Pr}(E)$ is lower). Thus, if one were to analyse the logical relations between the examples given here, one could say that the evidential result of the low specific heat of diamond better confirms the quantum postulate than Stokes's rule because we should assign a lower prior to this evidential result that seems to conflict with classical theory. Consequently, we need not appeal to old evidence or anomalous phenomena at all to explain this difference ${ }^{6}$ However, I would argue that this result actually bolsters the importance of the analysis given here, in the following sense.

Note that Hartmann \& Fitelson's solution to POE aims essentially to provide an accurate description of how old evidence for a hypothesis can affect an agent's credence in that hypothesis. By introducing extrasystematic statements such as $X$ and $Y$, Hartmann \& Fitelson explicitly consider an agent's credal state at a time when they are learning something about the relation between a theory and previously known observations because the logical relations that exist between theory and evidence were not accessible before this point. However, it is desirable that a descriptive account of an agent's reasoning at this point correspond to results in Bayesian confirmation that match with our methodological intuitions. What I have shown

\footnotetext{
${ }^{6} \mathrm{I}$ am grateful to an anonymous reviewer for raising this point.
} 
Copyright Philosophy of Science 2017

Preprint (not copyedited or formatted)

Please use DOI when citing or quoting

is that the framework Hartmann \& Fitelson give for explicating the role of old evidence when $\operatorname{Pr}(E)=1$ corresponds to the intuitively plausible result of a purely logical analysis of the relations between the hypothesis and evidential phenomena. Given that the historical case I have presented is exactly one in which $\operatorname{Pr}(E)=1$ for each of the phenomena in question, this analysis provides an account of how scientists may have made the judgments they did in a way that corresponds to the logical relation.

\section{Conclusion}

I have investigated Hartmann \& Fitelson's suggested solution to the problem of old evidence and whether it can explicate at least one important episode in the history of science, namely, the ability of the quantum postulate to account for several previously unexplained phenomena in the early developmental stages of quantum theory. I claimed that while their solution provides a good starting framework, an analysis of the historical facts compels us to introduce two new formal assumptions, namely that $a-c>b-d$ holds, and that $\operatorname{Pr}(Y)$ and $\operatorname{Pr}(X)$ are independent. Doing so enables the solution to differentiate between cases of old evidence with varying levels of support, depending on how anomalous the phenomenon in question is. I have argued for the idea that those assumptions are reasonable in the context under discussion. This analysis yielded an important conclusion: the given framework explicates the role of old evidence in a way that corresponds to the generally accepted Bayesian result that an unexpected consequence of a theory better supports that theory than a less surprising consequence. We thus see that not only can the given solution genuinely explicate an important example of old evidence in science, but that it can also explicate a desirable feature of Bayesian confirmation that has previously been problematic in that context.

\section{References}

Bohr, Niels. 1913. "On the Constitution of Atoms and Molecules, Part I." Philosophical Magazine 26:1-25.

Christensen, David. 1999. "Measuring Confirmation." The Journal of Philosophy 96 (9): $437-461$.

Eells, Ellery. 1990. "Bayesian Problems of Old Evidence.” In Scientific Theories, edited by C. Wade Savage, 205-223. Minneapolis, USA: University of Minnesota Press.

Einstein, Albert. 1905. "Ueber einen die Erzeugung und Verwandlung des Lichtes betreffenden heuristischen Gesichtspunkt." Annalen der Physik 17:132-148. Translated by D. ter Haar in The Old Quantum Theory 1967, 91-107. 
Copyright Philosophy of Science 2017

Preprint (not copyedited or formatted)

Please use DOI when citing or quoting

. 1907. "Die Plancksche Theorie der Strahlung und die Theorie der spezifischen Waerme.” Annalen der Physik 22:180-190.

- 1907/1989. "Planck's Theory of Radiation and the Theory of Specific Heat." In The Collected Papers of Albert Einstein, Volume 2: The Swiss Years: Writings, 1900-1909 (English translation supplement), 214-224. Translated by Anna Beck. Original work published 1907.

Garber, Daniel. 1983. "Old Evidence and Logical Omniscience in Bayesian Confirmation Theory." In Testing Scientific Theories: Midwest Studies in the Philosophy of Science, Vol. X, edited by John Earman, 99-131. Minneapolis, USA: University of Minnesota Press.

Hartmann, Stephan, and Branden Fitelson. 2015. "A New Garber-Style Solution to the Problem of Old Evidence." Philosophy of Science 82 (4): 712-717.

Howson, Colin. 1991. “The 'Old Evidence' Problem.” The British Journal for the Philosophy of Science 42 (4): 547-555.

Jammer, Max. 1966. The Conceptual Development of Quantum Mechanics. United States of America: McGraw-Hill Inc.

Jeffrey, Richard C. 1983. "Bayesianism with a Human Face." In Testing Scientific Theories: Minnesota Studies in the Philosophy of Science, Vol. 10, edited by John Earman, 133-156. Minneapolis, USA: University of Minnesota Press.

Klein, Martin J. 1965. "Einstein, Specific Heats, and the Early Quantum Theory." Science 148 (3667): 173-180.

Nernst, Walther. 1910. "Sur les chaleurs spécifiques aux basses températures et le développement de la thermodynamique." Journal de Physique Théorique et Appliquée 9:721-749.

—. 1911. "Zur Theorie der spezifischen Wärme und über die Anwendung der Lehre von den Energiequanten auf physikalisch-chemische Fragen überhaupt." Zeitschrift für Elektrochemie 17:265-275.

William Thomson, Lord Kelvin. 1901. "Nineteenth Century Clouds over the Dynamical Theory of Heat and Light." In Baltimore Lectures on Molecular Dynamics and the Wave Theory of Light, 486-527. London: C.J. Clay and Sons, Cambridge University Press Warehouse. Also in Philosophical Magazine 2, (1-40) 1901. 